J. Austral. Math. Soc. (Series A) 45 (1988), 78-82

\title{
HARDY-LITTLEWOOD MAXIMAL FUNCTIONS ON SOME SOLVABLE LIE GROUPS
}

\author{
G. GAUdRY, S. GIULINI, A. HULANICKI, and A. M. MANTERo
}

(Received 3 December 1986)

Communicated by J. F. Price

Dedicated to Robert Edwards in recognition of
25 years' distinguished contribution to mathematics in Australia,
on the occasion of his retirement

\begin{abstract}
Let $N$ be a nilpotent simply connected Lie group, and $A$ a commutative connected $d$-dimensional Lie group of automorphisms of $N$ which correspond to semisimple endomorphisms of the Lie algebra of $N$ with positive eigenvalues. Form the split extension $S=N \times A \cong N \times$ a, a being the Lie algebra of $A$. We consider a family of "rectangles" $B_{r}$ in $S$, parameterized by $r>0$, such that the measure of $B_{r}$ behaves asymptotically as a fixed power of $r$. One can construct the Hardy-Littlewood maximal function operator $f \rightarrow M_{f}$ relative to left translates of the family $\left\{B_{r}\right\}$. We prove that $M$ is of weak type $(1,1)$. This complements a result of J.-O. Strömberg concerning maximal functions defined relative to hyperbolic balls in a symmetric space.
\end{abstract}

1980 Mathematics subject classification (Amer. Math. Soc.): primary 43 A 80, 22 E 30; secondary 42 B 25.

Let $G$ be a semi-simple connected non-compact Lie group with finite center and let $G=N A K$ be the Iwasawa decomposition of $G$. Let $S=G / K$ be the noncompact symmetric space. $N A$ acts on $S$ simply transitively and so there is a natural identification of the group $N A$ (the group of translations of $S$ ) and $S$. We write

$$
S=N A \text {. }
$$

This research was supported by the Australian Research Grants Scheme and the Flinders University Visiting Fellowship Scheme.

(C) 1988 Australian Mathematical Society $0263-6115 / 88 \$ A 2.00+0.00$ 
The $G$-invariant metric $\rho$ on $S$ is thus a left-invariant metric on $S$ and the $G$-invariant measure on $S$ is the left invariant Haar measure $\mu_{l}$ on $S$. In this setting a theorem of J.-O. Strömberg [2] reads

THEOREM. Let

$$
\mathbf{B}_{r}=\{s \in S: \rho(s, e) \leq r\} .
$$

The maximal function $M f$ defined by

$$
M f(s)=\sup _{r>0} \mu_{l}\left(\mathbf{B}_{r}\right)^{-1} \int_{s \mathbf{B}_{r}} f\left(s^{\prime}\right) d \mu_{l}\left(s^{\prime}\right)
$$

is of weak type $(1,1)$.

The aim of this note is to show that a similar theorem is true for other families of balls $\left\{B_{r}\right\}_{r>0}$ on $S$ (not $K$-invariant any more) and as a matter of fact, the proof is very easy and straightforward. As a simple calculation shows, the balls we consider and the balls with respect to the hyperbolic metric on the upper half-plane (identified with the ' $a x+b$ '-group as above) are not comparable in measure, so Strömberg's result and ours are not simple consequences of each other.

The setting of our theorem is as follows.

Let $N$ be a nilpotent simply connected Lie group. Let $A$ be a commutative connected $d$-dimensional Lie group of automorphisms of $N$ which (as linear transformations on $\mathbf{n}$ ) are semi-simple with positive eigenvalues. We write $A=\left\{e^{t}: t \in \mathbf{a}\right\}, N \ni x \rightarrow e^{t} x \in N$ being the action of $A$ on $N$. We then have $e^{t} \cdot e^{t^{\prime}} x=e^{t+t^{\prime}} x$.

Let

$$
N \ni x \rightarrow|x| \in \mathbf{R}^{+}
$$

be a continuous function on $N$ with the property that for some positive constants $c, C^{\prime}, Q$

$$
C r^{Q} \leq \text { measure }\{x:|x| \leq r\} \leq c^{\prime} r^{Q}
$$

for all $r>0$. For $t \in \mathbf{a}$ let $|t|=$ norm of the operator $t$ (acting on $\mathbf{n}$ ). We form the split extension of $N$ by $A$ :

$$
S=N A=N \times \mathbf{a}
$$

the multiplication being

$$
(x, t)\left(x^{\prime}, t^{\prime}\right)=\left(x+e^{-t} x^{\prime}, t+t^{\prime}\right) .
$$

Then the left and right invariant Haar measures on $S$ are,

$$
\begin{aligned}
d \mu_{l}(x, t) & =e^{-\operatorname{Tr} t} d x d t \\
d \mu_{r}(x, t) & =d x d t
\end{aligned}
$$

respectively. 
THEOREM. Let

$$
B_{\tau}=\{s=(x, t):|x| \leq r,|t| \leq r\} .
$$

The maximal function $\mathcal{M} f$ defined by

$$
\mathcal{M} f=\sup _{r>0} \mu_{l}\left(B_{r}\right)^{-1} \int_{s B_{r}} f\left(s^{\prime}\right) d \mu_{l}\left(s^{\prime}\right)
$$

is of weak type $(1,1)$.

The proof follows [2] but is much simpler. In fact the theorem is an immediate consequence of the following two propositions.

Let

$$
\begin{aligned}
& \mathcal{M}_{0} f(s)=\sup _{r \leq 1} \mu_{l}\left(B_{r}\right)^{-1} \int_{s B_{r}} f\left(s^{\prime}\right) d \mu_{l}\left(s^{\prime}\right), \\
& \mathcal{M}_{\infty} f(s)=\sup _{r \geq 1} \mu_{l}\left(B_{r}\right)^{-1} \int_{s B_{r}} f\left(s^{\prime}\right) d \mu_{l}\left(s^{\prime}\right) .
\end{aligned}
$$

PROPOSITION 1. $M_{0}$ is of weak type $(1,1)$.

Proposition 2. $\mathcal{M}_{\infty} f(s) \leq|f|+\check{\tau}(s)$, where $\check{\tau} \in L^{1}\left(S, \mu_{l}\right)$.

Proposition 1 follows from the following two easy lemmas.

LeMma 1. Let $E \subset S$ and $\mu_{l}(E)<+\infty$. Suppose

$$
E \subset \bigcup_{s \in \sigma} s B_{r(s)}, \quad r(s) \leq 1 .
$$

Then there exists a subset $\left\{s_{1}, s_{2}, \ldots\right\}$ of $\sigma$ such that if $B_{r_{j}}=B_{r\left(s_{j}\right)}$, then

$$
s_{i} B_{r_{i}} \cap s_{j} B_{r_{j}}=\varnothing \quad \text { for } i \neq j
$$

and

$$
E \subset \bigcup_{j} s_{j} B_{r_{j}} B_{2 r_{j}}^{-1} B_{2 r_{j}}
$$

The proof is standard.

LEMMA 2. There is a constant $C$ such that

$$
\mu_{l}\left(B_{r} B_{2 r}^{-1} B_{2 r}\right) \leq C \mu_{l}\left(B_{r}\right)
$$

for all $r \leq 1$.

ProOF OF PROPOSITION 2. We have

$$
\mu_{l}\left(B_{r}\right) \geq C r^{Q} \int_{|t| \leq r} e^{-\operatorname{Tr} t} d t=C r^{Q}(s h r)^{d}
$$


Following J.-L. Clerc and E. M. Stein [1], see also [1], we get

$$
\varphi(x, t)= \begin{cases}\mu_{l}\left(B_{|x|}\right)^{-1} & \text { if }|x| \geq \max \{|t|, 1\}, \\ \mu_{l}\left(B_{|t|}\right)^{-1} & \text { if }|t| \geq \max \{|x|, 1\}, \\ 1 & \text { otherwise. }\end{cases}
$$

and we note that for a constant $C$

$$
\mu_{l}\left(B_{r}\right)^{-1} \chi_{B_{r}}(x, t) \leq C \varphi(x, t)
$$

for all $r \geq 1$, where $\chi_{E}$ denotes the indicator function of $E$. In fact, it suffices to verify (2) for

$$
r_{0}=\min \left\{r:(x, t) \in B_{r}\right\}
$$

and for $r_{0}(2)$ is obvious.

By (1), we have

$$
\varphi(x, t) \leq C\left(1+|x|^{Q}(s h|x|)^{d}+|t|^{Q}(s h|t|)^{d}\right)^{-1}=\tau(x, t) .
$$

Consequently, by (2),

$$
\begin{aligned}
\mathcal{M}_{\infty} f(s) & =\sup _{r \geq 1} \mu_{l}\left(B_{r}\right)^{-1} \int \chi_{s B_{r}}\left(s^{\prime}\right) f\left(s^{\prime}\right) d \mu_{l}\left(s^{\prime}\right) \\
& =\sup _{r \geq 1} \int \mu_{l}\left(B_{r}\right)^{-1} \chi_{B_{r}}\left(s^{\prime-1} s\right) f\left(s^{\prime}\right) d \mu_{l}\left(s^{\prime}\right) \\
& \leq|f| * \nsucc(x) .
\end{aligned}
$$

But clearly

$$
\int\left(1+|x|^{Q}(s h|x|)^{d}+|t|^{Q}(s h|t|)^{d}\right)^{-1} d t d x<+\infty
$$

i.e.

$$
\tau \in L^{1}\left(S, \mu_{r}\right)
$$

whence $\check{\tau} \in L^{1}\left(S, \mu_{l}\right)$, and the proof is complete.

REMARK. It would perhaps be interesting to know whether a similar result holds for riemannian balls with respect to some left-invariant riemannian metric on a solvable Lie group $S$. 


\section{References}

[1] J.-L. Clerc and E. M. Stein, ' $L^{p}$-multipliers for non-compact symmetric spaces', Proc. Nat. Acad. Sci. U.S.A 71 (1974), 3911-3912.

[2] Jan-Olov Strömberg, 'Weak type $L^{1}$ estimates for maximal functions on non-compact symmetric spaces', Ann. of Math. 114 (1981), 115-126.

School of Mathematical Sciences

Flinders University of South Australia

Bedford Park, South Australia 5042

Australia

Mathematics Institute

Wrocław University

Wroclaw

Poland
Dipartimento di Matematica "F. Enriques" Università di Milano

Milano

Italy

Istituto di Matematica

Università di Genova

Genova

Italy 